\title{
Probing signals with ZACZ for GPRonboard of unmanned aerial vehicle
}

\author{
Roman N. Ipanov, Aleksey A. Komarov \\ National Research University, Moscow Power Engineering Institute, Moscow, Russia
}

\begin{tabular}{ll}
\hline \hline Article Info & ABSTRACT \\
\cline { 2 - 3 } Article history: & $\begin{array}{l}\text { Modern ground penetrating radars (GPR), designed to determine the thickness } \\
\text { of ice or search for the occurrence of aquifers in arid regions of the Earth, are } \\
\text { installed either on helicopters or on the earth's surface. The use of a helicopter } \\
\text { Received Jul 4, 2020 } \\
\text { Revised Aug 7, 2020 } \\
\text { Accepted Aug 21, 2020 }\end{array}$ \\
$\begin{array}{l}\text { of a local nature. Modern GPRs mainly use video pulse probing sugnals and } \\
\text { probing signals with linear frequency modulation. These signals have } \\
\text { Keywords: }\end{array}$ & $\begin{array}{l}\text { correlation noise, which makes it difficult to obtain a high-quality radar image. } \\
\text { In this work, we propose to use a signal with a zero autocorrelation zone } \\
\text { (ZACZ) as a probing signal for GPR installed on an unmanned aerial vehicle. }\end{array}$ \\
$\begin{array}{l}\text { Ambiguity function } \\
\text { Autocorrelation function } \\
\text { Complementary sequences }\end{array}$ & $\begin{array}{l}\text { comparative analysis of the correlation characteristics of the synthesized signal } \\
\text { with the optimal phase-code shift keyed signal is carried out. }\end{array}$ \\
Pulse train &
\end{tabular}

This is an open access article under the CC BY-SA license.

Zero autocorrelation zone

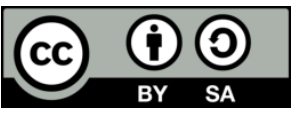

Corresponding Author:

Roman N. Ipanov

National Research University

Moscow Power Engineering Institute, Moscow, Russia

Email: iproman@ya.ru

\section{INTRODUCTION}

Modern ground penetrating radars (GPR) are capable of performing the following tasks: analyzing the soil during the construction of various objects [1, 2], monitoring road surfaces and railway tracks [3, 4], detecting various subsurface objects [5-7], solving archaeological problems [8,9], studying ice covers, etc. However, all these GPRs are installed either on helicopters [10-13], which perform sounding of glaciers and ice thickness over glacial lakes, or on the Earth's surface to detect and determine the depth of aquifers at a depth of 10 to $40 \mathrm{~m}$ [14-16].

GPRs mainly use video pulse probing signals $[17,18]$ and signals with linear frequency modulation [19-22]. In [23] for georadars installed on unmanned aerial vehicles (UAV), a probing signal with a zero autocorrelation zone (ZACZ) was proposed for georadar working in a quasi-continuous operation mode. These signals have correlation noise, which makes it difficult to obtain a high-quality radar image. In this work, we propose to use a signal with a ZACZ as a probing signal for GPR installed on an UAV. The objective of the paper is to synthesize a polyphase (p-phase) probing signal with a ZACZ consisting of p phase-code shift keyed pulses in the train, for a GPR onboard of unmanned aerial vehicle working in a pulsed operation mode.

\section{SYNTHESIS PROBING SIGNAL WITH ZACZ FOR GPR ONBOARD OF UNMANNED AERIAL VEHICLE}

Let us consider the polyphase PCSK-signal, which is a sequence (train) of $M$ pulses encoded with the ensemble of $M p$-ary sequences [24]. Each pulse with duration $T_{\mathrm{p}}$ consists of $N$ sub-pulses (discretes) with 
duration $T_{0}=T_{\mathrm{p}} / N$ each. The repetition period of the pulses is $T=Q T_{\mathrm{p}}=Q N T_{0}$, where $Q \geq 2$ is the offduty factor of the pulse train. The ensemble of $M p$-ary sequences can be set according to the matrix:

$$
\mathbf{A}_{M, N}=\left\|a_{i, n}\right\|_{i, n=1}^{M, N}, a_{i, n}=\exp \left(j \frac{2 \pi}{p} \tilde{a}_{i, n}\right)
$$

where $p>2$ is the prime integer number (the signal with $p=2$ is considered in [24]); $\tilde{\mathbf{A}}_{M, N}=\left\|\tilde{a}_{i, n}\right\|_{i, n=1}^{M, N}$, $\tilde{a}_{i, n}=0,1, \ldots, p-1$, is the matrix of $p$-ary code.

The complex envelope (CE) of the considered signal is as follows:

$$
\dot{u}(t)=\sum_{i=1}^{M} \sum_{n=1}^{N} a_{i, n} \dot{S}_{n}\left(t-(n-1) T_{0}-(i-1) T\right)
$$

where $\dot{S}_{n}\left(t-(n-1) T_{0}\right),(n-1) T_{0} \leq t<n T_{0}$, is the CE of the $n$-th discrete of the pulse.

If $\dot{S}_{1}(t)=\dot{S}_{2}(t)=\ldots=\dot{S}_{N}(t)$ and the rows of the matrix $\mathbf{A}_{M, N}$ (1) are complementary sequences, then expression (2) is the signal with the zero zone of the side lobes (SL) of autocorrelation function (ACF) $R(\tau)$ at $|\tau| \leq T_{\mathrm{p}}$ [25]. Such signal at $M=p$ and $\dot{S}_{n}(t)=S_{n}(t), n=1,2, \ldots, N$, was considered in [26, 27].

Let us consider that the coding matrix (1) at $M=p$ is the following block matrix:

$$
\mathbf{A}_{p, N}=\left(\begin{array}{llllll}
\mathbf{A}_{p, N / p}^{(1)} & \mathbf{A}_{p, N / p}^{(2)} & \ldots & \mathbf{A}_{p, N / p}^{(k)} & \ldots & \mathbf{A}_{p, N / p}^{(p)}
\end{array}\right)
$$

where sub-matrices are,

$$
\begin{aligned}
& \mathbf{A}_{p, N / p}^{(k)}=\left\|a_{i, n}^{(k)}\right\|_{i, n=1}^{p, N / p}, \quad k=1,2, \ldots, p \\
& \sum_{i=1}^{p} \sum_{n=1}^{N / p-m} a_{i, n+m}^{(k)} a_{i, n}^{(l)^{*}}=0 \\
& k, l=1,2, \ldots, p ; \\
& m=\left\{\begin{array}{l}
0,1, \ldots, N / p-1 \text { at } k \neq l ; \\
1,2,, \ldots, N / p-1 \text { at } k=l
\end{array}\right. \\
& \text { If } \quad \dot{S}_{(k-1) \frac{N}{p}+1}(t)=\ldots=\dot{S}_{k \frac{N}{p}}(t), k_{k=1,2, \ldots, p \text { and matrix (1) at } M=p \text { are of the form (3), then (2) is }}
\end{aligned}
$$
the signal with the zero zone of ACF SLs $R(\tau)$ at $|\tau| \leq T_{\mathrm{p}}[24,25,28]$.

Let us consider the PCSK signal (2) at $M=p$ encoded with the rows of the matrix (3), where the sub-matrixes $\mathbf{A}_{p, N / p}^{(k)}$ are the adjacent p-pairs of p-ary D-code [24, 26-29]. Then any two of the p submatrices $\mathbf{A}_{p, N / p}^{(k)}$ in expression (3) will be as follows:

$$
\mathbf{A}_{p, N / p}^{(k)}=\left(\begin{array}{c}
\mathbf{D}_{1, N / p}^{k_{1}} \\
\mathbf{D}_{1, N / p}^{k_{2}} \\
\vdots \\
\mathbf{D}_{1, N / p}^{k_{p}}
\end{array}\right) ; \quad \mathbf{A}_{p, N / p}^{(l)}=\left(\begin{array}{c}
\mathbf{D}_{1, N / p}^{l_{1}} \\
\mathbf{D}_{1, N / p}^{l_{2}} \\
\vdots \\
\mathbf{D}_{1, N / p}^{l_{p}}
\end{array}\right) ; \quad k, l=1,2, \ldots, p,
$$


where $\mathbf{D}_{1, N / p}^{k_{i}}$ and $\mathbf{D}_{1, N / p}^{k_{j}}, \quad \mathbf{D}_{1, N / p}^{l_{i}}$ and $\mathbf{D}_{1, N / p}^{l_{j}}$ at $i, j=1,2, \ldots, p, \quad i \neq j$, are p-pair sequences (p-pairs) [26, 27], and $\mathbf{D}_{1, N / p}^{k_{i}}$ and $\mathbf{D}_{1, N / p}^{l_{i}}$ are the adjacent sequences of p-ary D-code [26, 27, 29]; $k_{i}, l_{i}=1,2, \ldots, N / p$ are the numbers of sequences in p-ary D code; $N=p^{q+1}, q \geq 2$.

For $p$-pair sequences, the condition (4) is fulfilled at $k=l$, and for adjacent sequences of $p$-ary Dcode, the condition (4) is fulfilled at $k \neq l([24,26-29]$.

Let us call the PCSK-signal (2) encoded with the rows of the matrix $\mathbf{A}_{p, N}$ from (3) with submatrices from (5) a polyphase coherent complementary signal (CCS). Thus, the polyphase CCS is the signal with ZACZ at $|\tau| \leq T_{\mathrm{p}}$ with the CE of the discretes $\dot{S}_{n}(t)$ of each of p pulses of the train being equal to each other at $n=(k-1) N / p+1, \ldots, k N / p$, where $k=1,2, \ldots, p$ is the number of the pulse part with equal complex envelopes of discretes.

In accordance with the aforesaid, let us consider the polyphase CCS with additional frequency shift keying of the discretes of the pulses of train (CCS-FSK). Each discrete here consists of $\mathrm{b}$ bits with duration $T_{\mathrm{b}}=T_{0} / b$. Each of p CCS-FSK pulses consists of $N=p^{q+1}$ discretes and is divided into p parts with the number of discretes in each equal to $p^{q}$. The discretes of the first ${ }^{p-1}$ parts are manipulated by linear law, and the discretes of the last p-th part are manipulated by frequency so that to ensure the orthogonality of their bits with the bits of the discretes of the first ${ }^{p-1}$ parts. Figure 1 shows a variant of the FSK law of discretes within one CCS-FSK pulse at $p=3$ and $b=9$, the first discrete of the first part, the last discrete of the second part and the last discrete of the third part of the pulse of the train.

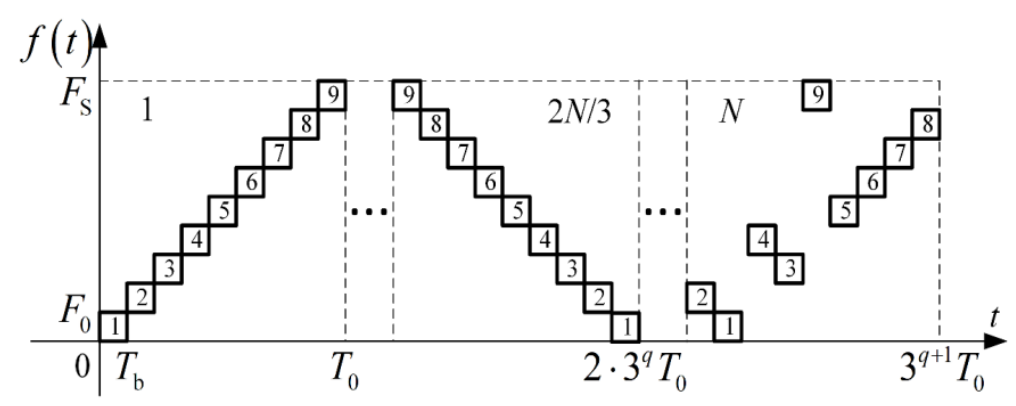

Figure 1. FSK law of the discrete within the CCS pulse at $p=3$ and $b=9$

The law of frequency variation within r-bit with account for Figure 1 is as follows:

$$
f_{r}(t)=\left\{\begin{array}{l}
\left(b^{\left\langle\lfloor-1\rfloor_{b p} q\right\rangle_{2}}+(-1)^{\lfloor-1\rfloor_{b p} q}\langle r-1\rangle_{b}\right) F_{0}, \\
\text { at } r=1, \ldots, b(p-1) N / p ; \\
\left(\kappa_{\langle r-1\rangle_{b}+1}+1\right) F_{0}, \\
\text { at } r=b(p-1) N / p+1, \ldots, b N,
\end{array}\right.
$$

where $\langle y\rangle_{M}$ is number y by module $\mathrm{M} ;{ }^{\lfloor y\rfloor_{M}}$ is the integer part of number $y / M ; r=1,2, . ., b N$ is the bit number in the CCS-FSK pulse; $F_{\mathrm{S}}=b F_{0}=b / T_{\mathrm{b}}=b^{2} / T_{0}$ is the CCS- FSK spectrum width; $\kappa_{v}=0,1, \ldots, b-1$ 
is the symbol of frequency code sequence (FCS) $\left\{\kappa_{v}\right\}_{v=1}^{b}$, which is orthogonal to two linear FCSs (for the case shown in Figure $1\left\{\kappa_{v}\right\}_{v=1}^{9}=1,0,3,2,8,4,5,6,7$ ).

Thus, CE (2) of CCS-FSK is as follows:

$$
\begin{gathered}
\dot{u}(t)=\sum_{i=1}^{p}\left[\sum_{r=1}^{b(p-1) N / p} S_{\lfloor r-1\rfloor_{b p} q}\left(t-(r-1) T_{\mathrm{b}}-(i-1) T\right) \times\right. \\
\times \exp \left\{\mathrm { j } \frac { 2 \pi } { p } \left\{p\left[b^{\left\langle\lfloor r-1\rfloor_{b p} q\right\rangle_{2}}+(-1)^{\lfloor r-1\rfloor_{b p} q}\langle r-1\rangle_{b}\right] \frac{t}{T_{\mathrm{b}}}+\tilde{a}_{\left.\left.i,\lfloor r-1\rfloor_{b}+1\right)\right\}+}\right.\right. \\
+\sum_{r=b(p-1) N / p+1}^{b N} S_{p-1}\left(t-(r-1) T_{\mathrm{b}}-(i-1) T\right) \times \\
\left.\times \exp \left\{\mathrm{j} \frac{2 \pi}{p}\left(p\left[\kappa_{\langle r-1\rangle_{b}+1}+1\right] \frac{t}{T_{\mathrm{b}}}+\tilde{a}_{i,\lfloor r-1\rfloor_{b}+1}\right)\right\}\right]
\end{gathered}
$$

where $S_{n}(t)=S_{k-1}(t), \quad n=(k-1) N / p+1, \ldots, k N / p, \quad k=1,2, \ldots, p$, is the envelope of the n-th discrete of CCS-FSK pulse; $\tilde{a}_{i, n}$ is the symbol of p-ary code defined by expressions (1), (3) and (5).

\section{ANALYSIS OF THE CORRELATION CHARACTERISTICS OF THE PROBING PCSK- \\ SIGNAL WITH ZACZ}

Let us carry out a comparative analysis of the correlation characteristics of polyphase CCS without modulation of discretes (further we will denote it as CCS) [26, 27] with correlation characteristics of polyphase CCS-FSK at $p=3$. To compare the relative level of the SLs of these two signals, it is necessary to provide an identical level of the main lobes of their ACFs, which is equal to the number of discretes (bits) in the three pulses of the train. Let us consider the CCS with the number of discretes in the pulse $N=3^{7}$ and the CCS-FSK with $N=3^{5}$ and with the number of bits in the discrete $b=9$. Both signals have an identical off-duty factor $Q=2$. Thus, the level of the main lobe of ACF of both signals is 6561 .

It is known [30] that signals that have the minimal level of the maximal SL (MSL) of the aperiodic $\mathrm{ACF}$, i.e. ones for which the criterion:

$$
R_{\max }=\max _{1 \leq 1 m \leq N-1}\left\{\left|R_{m}\right|\right\} \Rightarrow \min
$$

is met, where $R_{m}=1 / N \sum_{n=1}^{N-m} a_{n}^{*} a_{n+m}, m=0, \pm 1, \ldots, \pm(N-1)$, are called optimal discrete sequences (signals) with a length of $\mathrm{N}$.

PCSK-signals with the relative level of the MSL $R_{\max }=1 / N$ that reach boundary (8) are called Barker codes in the literature. Binary Barker codes exist only for the lengths $\mathrm{N}=2,3,4,5,7,11,13$. There are no binary Barker codes for the other odd lengths, and for even within the range of $13<N<2 \cdot 10^{30}$ [31].

In building the optimal pulse sequences, current researches are aimed at two goals:

a) building the binary sequences with the least possible values $R_{\max }$ (8);

b) building the multiphase Barker sequences.

So far, no regular way of building the sequences optimal by criterion (8) is known. That is why, computer search algorithms are utilized when building the optimal binary sequences, and numeric optimization methods are used when building the optimal multiphase sequences.

By now, binary sequences with level 5 of the MSL of the unnormalized ACF have been found for all the lengths of sequences from the range $83 \leq N \leq 105$ [32]. If the length $\mathrm{N}$ is further increased, the computational complexity of search algorithms becomes extremely high for modern computers. That is why, the following approach is used. At the first stage, periodic binary sequences with a two-, three-, and-so-on- 
level periodic ACF are synthesized. At the second stage, cyclically shifted sequences are built on the basis on the source one, and the sequences that have the minimal value of the ACF MSL are selected from among them. This approach permits synthesizing very long binary sequences with the normalized aperiodic ACF's SL level that does not exceed $1 / \sqrt{N}$ [30]. Such sequences include M-sequences, which are the most frequently used in radars as a probing signal.

The longest, for today, multiphase Barker sequences were obtained in study [33] for $65 \leq N \leq 70$, $N=72, N=76, N=77$. At that, as the lengths of the sequences increased, the phase alphabet from the range 0 to $2 \pi$ increases infinitely. Study [34] argues that p-phase Barker codes must exist for large lengths $N$, provided that the phases are graded from $p \geq N$.

Also, multiphase Frank signals [35], the periodic ACF of which has zero side lobes, are known. The length of the Frank sequence is $N=p^{2}$, where $\mathrm{p}$ is the quantity of the phases. The aperiodic ACF has the relative level of the MSL at $N \geq 9 \quad R_{\max } \approx 1 /(3 \sqrt{N})$. The ambiguity function (AF) of a Frank signal is close to the AF of a signal with the linear frequency modulation, i.e. it has a crest.

Thus, it is reasonable to use a truncated M-sequence for the comparative analysis of the correlational characteristics, since at the unlimited length of its period $N_{\mathrm{M}}$ it is optimal and has a button AF with the relative level of MSL approximately equal to $1 / \sqrt{N_{\mathrm{M}}}$ in the delay-frequency plane.

Let us carry out a comparative analysis of the correlation characteristics of the CCS and the CCSFSK with a PCSK-signal manipulated by phase using a ternary M-sequence (further we will denote it as MS) with the period $N_{\mathrm{M}}=3^{8}-1$. To equalize the levels of the main lobes of the ACF of CCS and the MS let us add an element from the adjacent period to its period, i.e. the number of discretes in the MS pulse will be $N=N_{\mathrm{M}}+1=6561$

Table 1 provides the indices of correlation characteristics of the CCS, CCS-FSK and MS for three sections of their AF $R(\tau, F)$ with the planes $F=0, F=0.3 \Delta F$ and $F=0.5 \Delta F$, where $\Delta F=1 /\left(p Q T_{\mathrm{p}}\right)$ is the width of the main lobe of the section of AF with the plane $\tau=0 \quad R(0, F)$ at $-3 \mathrm{~dB}$. In Table $1 R_{\max }$ and $R_{\mathrm{z} \text { max }}$ are the relative levels of the MSL of ACF outside of the ZACZ and in the ZACZ respectively, $R_{\mathrm{rms}}$ and $R_{\mathrm{z} \text { rms }}$ are the relative root-mean-square levels (rms) of the SL of ACF outside of the ZACZ and in the ZACZ respectively. Table 1 shows that the level of all SLs of CCS-FSK ACF is less than the level of the SLs of CCS ACF by the rms of SLs more than by $10 \mathrm{~dB}$. The rms of the SLs of CCS-FSK ACF is more than by $10 \mathrm{~dB}$ less than the rms of the SLs of MS ACF.

Table 1. Indices of correlation characteristics of the CCS, CCS-FSK and MS

\begin{tabular}{|c|c|c|c|c|}
\hline \multicolumn{2}{|c|}{$F$} & 0 & $0.3 \Delta F$ & $0.5 \Delta F$ \\
\hline \multirow{3}{*}{$R_{\max }, \mathrm{dB}$} & CCS & -25.5 & -23.0 & -21.5 \\
\hline & CCS-FSK & -28.9 & -27.8 & -27.4 \\
\hline & MS & -39.6 & -38.8 & -36.9 \\
\hline \multirow{3}{*}{$R_{\mathrm{rms}}, \mathrm{dB}$} & CCS & -45.7 & -45.7 & -45.6 \\
\hline & CCS-FSK & -55.4 & -55.3 & -55.2 \\
\hline & MS & -45.9 & -44.7 & -43.4 \\
\hline \multirow{2}{*}{$R_{\mathrm{z} \max }, \mathrm{dB}$} & CCS & -328.0 & -21.3 & -16.9 \\
\hline & CCS-FSK & -327.0 & -29.5 & -25.2 \\
\hline \multirow{2}{*}{$R_{\mathrm{zrms}}, \mathrm{dB}$} & CCS & -348.8 & -48.0 & -44.4 \\
\hline & CCS-FSK & -357.6 & -57.8 & -54.1 \\
\hline
\end{tabular}

Figure 2 shows the zero zones of the ACF SLs $R(\tau)$ at $\tau \leq b N T_{\mathrm{b}}$ (ZACZ) of the considered signals of the CCS and of the CCS-FSK, as well as a part of the MS ACF in case of a mismatch by frequency at $F=0.3 \Delta F$. The time scale here is shown as the number of bits, and for the CCS and MS it is assumed that $T_{0}=T_{\mathrm{b}}$. Figure 2 shows that in case of a mismatch by frequency at $F=0.3 \Delta F$, the level of the majority of the SLs of ACF of the CCS-FSK is less than the level of the SLs of MS, which is in accordance with the data provided in Table 1. 


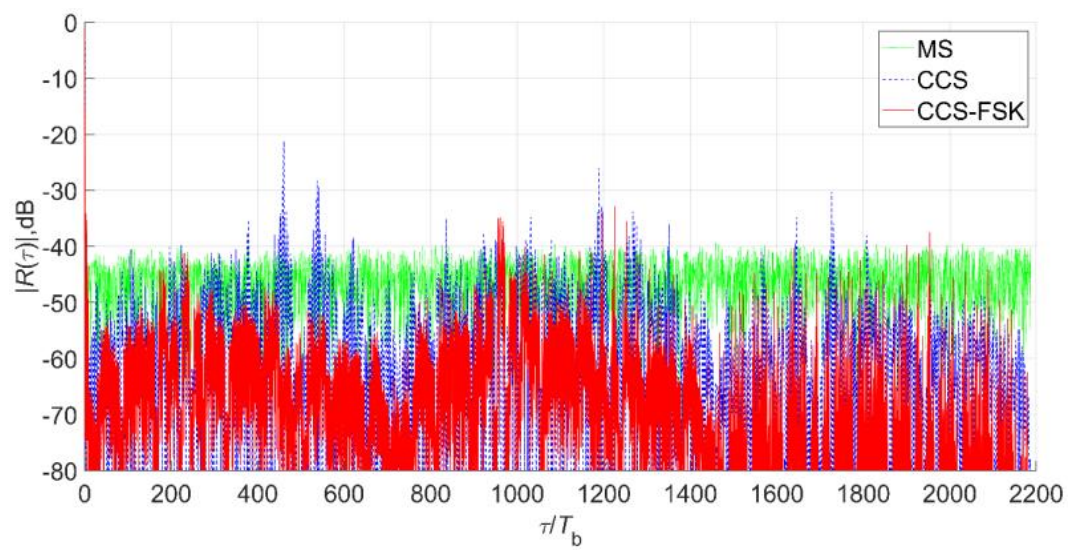

Figure 2. ZACZ of CCS, CCS-FSK and ACF of MS at $F=0.3 \Delta F$

\section{CONCLUSION}

In the work we have proposed a UAV-based GPR for the rapid search for shallow aquifers. For a GPR onboard of a UAV, a polyphase (p-phase where $\mathrm{p}$ is a prime number) probing signal with ZACZ has been synthesized, which signal represents a sequence of p PCSK-pulses coded by complementary sequences of the p-ary D-code with additional frequency shift-keying of the sub-pulses of pulses. A comparative analysis of the correlation characteristics of the synthesized three-phase signal with a signal without modulation of discretes and with a PCSK-signal coded by ternary MS has been performed. The analysis has revealed that in case of a mismatch by Doppler frequency, the rms level of the SLs of ACF of the synthesized signal is more than by $10 \mathrm{~dB}$ less than the rms level of the SLs of the ACF of the PCSK-signal coded by MS.

\section{ACKNOWLEDGEMENTS}

The reported study was funded by RFBR and MCESSM according to the research project № 19-57-44001.

\section{REFERENCES}

[1] M. Pipan, E. Forte, G. Dal Moro, M. Sugan and I. Finetti, "Integrated vertical radar profiles (VRP) and multi-fold GPR for site characterization," in $2^{\text {nd }}$ Int. Workshop on Advanced Ground Penetrating Radar, Delft, Netherlands, 2003, DOI: 10.1109/AGPR.2003.1207304.

[2] M. Bimpas, A. Amditis and N. Uzunoglu, "Design and implementation of an integrated high resolution imaging ground penetrating radar for water pipeline rehabilitation," in $11^{\text {th }}$ Int. Radar Symposium, Vilnius, Lithuania, 2010.

[3] L. Yi, L. Zou and M. Sato, "A simplified velocity estimation method for monitoring the damaged pavement by a multistatic GPR system YAKUMO," in $201817^{\text {th }}$ Int. Conf. on Ground Penetrating Radar (GPR), Rapperswil, Switzerland, 2018, DOI: 10.1109/ICGPR.2018.8441531.

[4] D. Pasculli, A. Natali, W. Salvatore, F. Morelli and D. Morandi, "Investigation of reinforced concrete bridges by using a dual-polarized high-frequency GPR," in $201817^{\text {th }}$ Int. Conf. on Ground Penetrating Radar (GPR), Rapperswil, Switzerland, 2018, DOI: 10.1109/ICGPR.2018.8441633.

[5] H. M. Jol and A. Albrecht, "Searching for submerged lumber with ground penetrating radar: Rib Lake, Wisconsin, USA," in $10^{\text {th }}$ Int. Conf. on Grounds Penetrating Radar (GPR), Delft, Netherlands, pp. 601-604, 2004.

[6] W. Kofman, "Radar techniques to study subsurfaces and interiors of the solar system objects," in $201219^{\text {th }}$ Int. Conf. on Microwaves, Radar \& Wireless Communications, Warsaw, Poland, pp. 409-412, 2012. DOI: 10.1109/MIKON.2012.6233605.

[7] J. C. Ralston and A. D. Strange, "An industrial application of ground penetrating radar for coal mining horizon sensing," in 2015 Int. Symposium on Antennas and Propagation (ISAP), Hobart, TAS, Australia, pp. 1-4, 2015.

[8] R. D. Evans, R. Morrow and J. Nash, "The use of ground penetrating radar to investigate a Churchyard Burial Plot," in $15^{\text {th }}$ Int. Conf. on Ground Penetrating Radar, Brussels, Belgium, pp. 45-49, 2014. DOI: 10.1109/ICGPR.2014.6970382.

[9] C. M. de Jong van Coevorden et al., "GPR survey at the archaeological roman site of Ciavieja, El Ejido (Spain)," in $20116^{\text {th }}$ Int. Workshop on Advanced Ground Penetrating Radar (IWAGPR), Aachen, Germany, pp. 1-4, 2011, DOI: 10.1109/IWAGPR.2011.5963848.

[10] G. J. Church, A. Bauder, M. Grab, S. Hellmann and H. Maurer, "High-resolution helicopter-borne ground penetrating radar survey to determine glacier base topography and the outlook of a proglacial lake," in $201817^{\text {th }}$ Int. Conf. on Ground Penetrating Radar (GPR), 2018, DOI: 10.1109/ICGPR.2018.8441598. 
[11] M. Grab, A. Bauder, F. Ammann, et al., "Ice volume estimates of Swiss glaciers using helicopter-borne GPR - an example from the Glacier de la Plaine Morte," in $201817^{\text {th }}$ Int. Conf. on Ground Penetrating Radar (GPR), Rapperswil, Switzerland, 2018, DOI: 10.1109/ICGPR.2018.8441613.

[12] L. Langhammer, L. Rabenstein, A. Bauder, et al., "Development of a novel dual-polarization helicopter-borne GPR system," in $201817^{\text {th }}$ Int. Conf. on Ground Penetrating Radar (GPR), 2018, DOI: 10.1109/ICGPR.2018.8441574.

[13] Bauder, G. Mazzotti, C. Berger, L. Langhammer, N. Griessinger and T. Jonas, "Winter Accumulation Measurements on Alpine Glaciers using Ground Penetrating Radar," in $201817^{\text {th }}$ Int. Conf. on Ground Penetrating Radar (GPR), Rapperswil, Switzerland, 2018, DOI: 10.1109/ICGPR.2018.8441559.

[14] K. Maheswari, P. Senthil Kumar, D. Mysaiah, K. Ratnamala, M. Sri Hari Rao and T. Seshunarayana, "Ground Penetrating Radar for Groundwater Exploration in Granitic Terrains: A Case Study from Hyderabad," Journal geological society of India, vol. 81, pp. 781-790, Jun. 2013, DOI: 10.1007/s12594-013-0103-x.

[15] Sebastian Kowalczyk, Anna Lejzerowicz and Beata Kowalczyk, "Groundwater table level changes based on ground penetrating radar images: a case study," in $201817^{\text {th }}$ Int. Conf. on Ground Penetrating Radar (GPR), Rapperswil, Switzerland, 2018, DOI: 10.1109/ICGPR.2018.8441628.

[16] J. Igel, T. Günther and M. Kuntzer, "Ground-penetrating radar insight into a coastal aquifer: the freshwater lens of Borkum Island," Hydrology and Earth System Sciences, vol. 17, no. 2, pp. 519-531, Feb. 2013, DOI: 10.5194/hess17-519-2013.

[17] L. Yu. Astanin and A. A. Kostylev, "Ultra-wideband Radar Measurements Systems, IEE Radar, Sonar, Navigation and Avionics Series", London: IEE Books, vol. 7, 1997.

[18] D. J. Daniels, "Resolution of ultra-wideband radar signals," IEE Proc. - Radar, Sonar and Navig., vol. 146, no. 4, pp. 189-194, Aug. 1999, DOI: 10.1049/ip-rsn:19990489.

[19] D. J. Daniels, "Surface Penetrating Radar, IEE Radar, Sonar, Navigation and Avionics Series," London: IEE Books, vol. 6, 1996.

[20] J. Jendo, M. Pasternak, "Synthetic Wideband Waveform for Ground Penetrating Radar Application," in 2019 Signal Processing Symposium (SPSympo), Krakow, Poland, 2019, DOI: 10.1109/SPS.2019.8881991.

[21] P. T. W. Wong, W. W. L. Lai, M. Sato, "Time-frequency spectral analysis of step frequency continuous wave and impulse ground penetrating radar," in $201616^{\text {th }}$ Int. Conf. on Ground Penetrating Radar (GPR), Hong Kong, China, 2016, DOI: 10.1109/ICGPR.2016.7572694.

[22] V. G. Sugak, A. V. Bukin, N. G. Reznichenko, Ali Djadooei, "Forward Looking ground penetrating radar with synthetic antenna aperture for buried explosive hazards detection," in $20169^{\text {th }}$ Int. Kharkiv Symposium on Physics and Engineering of Microwaves, Millimeter and Submillimeter Waves (MSMW), Kharkiv, Ukraine, 2016, DOI: 10.1109/MSMW.2016.7538068.

[23] R. N. Ipanov, A. A. Komarov, N. D. Luchkovskiy, "Phase-code shift keyed probing signals with zero autocorrelation zone for ground penetrating radar onboard of unmanned aerial vehicle," in Proc. 2020 Int. Youth Conf. on Radio Electronics, Electrical and Power Engineering (REEPE), Moscow, Russian Federation, 2020, DOI: 10.1109/REEPE49198.2020.9059237.

[24] R. N. Ipanov, A. A. Komarov, A. P. Klimova, "Phase-Code Shift Keyed Probing Signals with Discrete Linear Frequency Shift Keying and Zero Autocorrelation Zone," in 2019 Int. Conf. on Engineering and Telecommunication (EnT), 2019, DOI: 10.1109/EnT47717.2019.9030566.

[25] D. Chebanov and G. Lu, "Removing autocorrelation sidelobes of phase-coded waveforms," in IEEE Radar Conf., Washington, DC, USA, 2010, DOI: 10.1109/RADAR.2010.5494391.

[26] R. N Ipanov, "Polyphase Radar Signals with ZACZ Based on $p$-Pairs D-Code Sequences and Their Compression Algorithm," HTE Infocommunications Journal, vol. 11, no. 3, pp. 21-27, Nov. 2019, DOI: 10.36244/ICJ.2019.3.4.

[27] I. Baskakov, R. N. Ipanov, A. A. Komarov, "The Use of Phase-shift Keyed Signals with a Zero Autocorrelation Zone in a Multi-position Radar System for Searching and Detecting of Space Debris Objects," in 2019 PhotonIcs \& Electromagnetics Research Symposium (PIERS), 2019, DOI: 10.1109/PIERS-Spring46901.2019.9017759.

[28] R. N. Ipanov, "Signals with zero autocorrelation zone for the synthesised aperture radar," IET Electronics Letters, vol. 55, no. 19, pp. 10631065, Sep. 2019, DOI: 10.1049/el.2019.1918.

[29] R. N. Ipanov, "Pulsed Pha-se-Shift Keyed Signals with Zero Autocorrelation Zone," Journal of Communications Technology and Electronics, vol. 63, no. 8, pp. 895-901, Aug. 2018, DOI: 10.1134/S1064226918080077.

[30] V. P. Ipatov, "Spread Spectrum and CDMA, Principles and Applications", Hoboken, NJ, USA: Wiley, 2005.

[31] M. J. Mossinghoff, "Wieferich pairs and Barker sequences," Designs, Codes and Cryptography, vol. 53, no. 3, pp. 149-163, 2009, DOI: 10.1007/s10623-009-9301-3.

[32] C. J. Nunn, G. E. Coxson, "Best-known autocorrelation peak sidelobe levels for binary codes of length 71 to 105 ," IEEE Trans. Aerosp. Electron. Syst., vol. 44, no. 1, pp. 392-395, May 2008, DOI: 10.1109/TAES.2008.4517015.

[33] C. J. Nunn, G. E. Coxson, "Polyphase pulse compression codes with optimal peak and integrated sidelobes," IEEE Trans. Aerosp. Electron. Syst., vol. 45, no. 2, pp. 775-781, Jun 2009, DOI: 10.1109/TAES.2009.5089560.

[34] L. Ein-Dor, I. Kanter, W. Kinzel, "Low autocorrelated multiphase sequences," Physical Review E, vol. 65, no. 2, pp. 020102/1-020102/4, 2008, DOI: 10.1103/PhysRevE.65.020102.

[35] R. L. Frank, "Polyphase Codes with Good Nonperiodic Correlation Properties," IEEE Trans. Inf. Theory, vol. 9, no. 1, pp. 43-45, Jan 1963, DOI: 10.1109/TIT.1963.1057798. 


\section{BIOGRAPHIES OF AUTHORS}

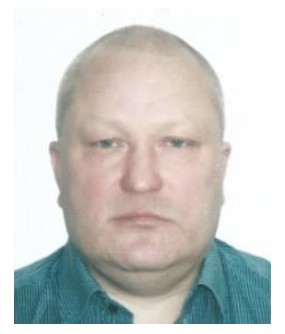

Roman N. Ipanov was born in Kishinev City, Moldova, USSR, in 1974. In 1999 he graduated in the specialty radio-electronic systems the Military Space Academy named after A. F. Mozhaisky, Saint Petersburg. He received the Ph.D. in the specialty radiolocation and radio navigation at the Military Space Academy named after AF Mozhaisky in 2004. From 2004 to 2010 he was a lecturer and a senior lecturer in the department of radiolocation at the Military Space Academy named after AF Mozhaisky. From 2013 to 2018 he worked as a senior researcher in the Federal Government Institution Scientific and Production Association Special Equipment and Telecoms of the Ministry of Internal Affairs of the Russian Federation, Moscow. Currently working as an associate professor at the Department of Radio Engineering Devices and Antenna Systems at the Moscow Power Engineering Institute, studying in doctoral studies. He is the author of more than 60 scientific papers and three inventions. Research interests include the synthesis and digital processing of radar signals.

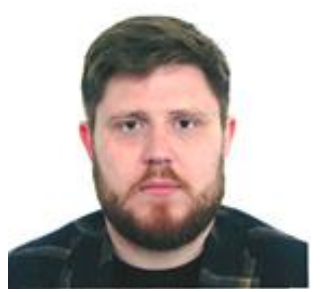

Aleksey A. Komarov was born in Ramenskoe, Moscow region, USSR, in 1988. He received the B.S., M.S., and Ph.D degrees in 2009, 2011 and 2013 respectively from National Research University "Moscow Power Engineering Institute". He is now the head of the Department of Radioengineering Devices and Antenna Systems at National Research University "Moscow Power Engineering Institute". His research interests are radiowave propagation, computational electromagnetics, remote sensing, radar imaging, and signal processing. 